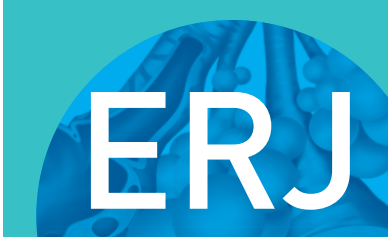

open research
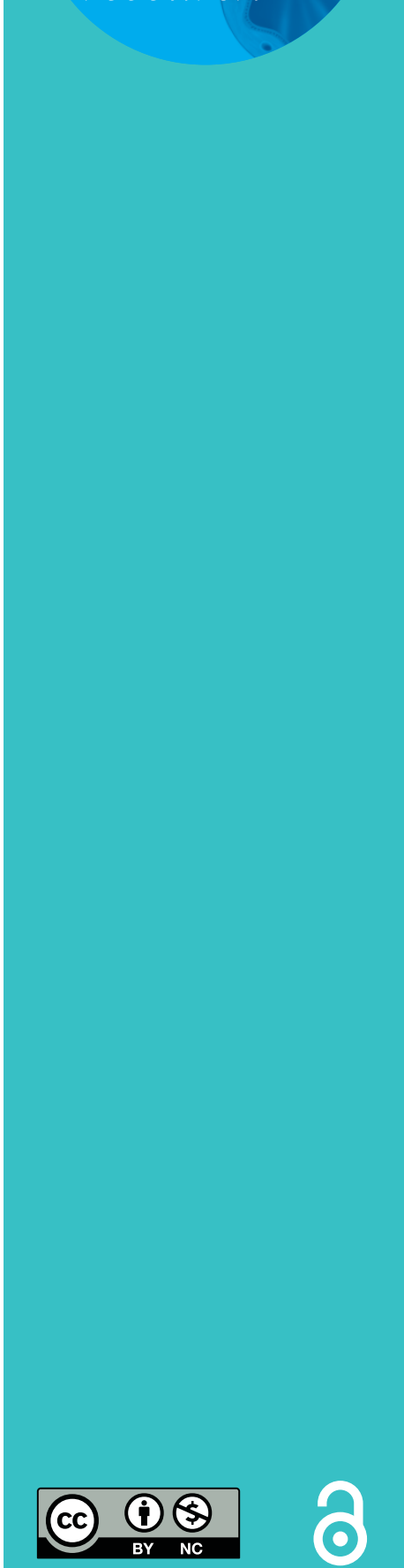

\section{Diaphragmatic motion recorded by M-mode ultrasonography: limits of normality}

\author{
Alain Boussuges ${ }^{1}$, Julie Finance $\mathbb{C}^{2}$, Guillaume Chaumet $^{3}$ and Fabienne Brégeon ${ }^{2}$
}

Affiliations: ${ }^{1}$ Institut de Recherche Biomédicale des Armées (IRBA), ERRSO, Toulon and Center for Cardiovascular and Nutrition Research (C2VN), Aix Marseille Université, INSERM, INRAE, Marseille, France. ${ }^{2}$ Service d'Explorations Fonctionnelles Respiratoires, CHU Nord, Assistance Publique des Hôpitaux de Marseille et Aix Marseille Univ, IRD, APHM, MEPHI, IHU-Méditerranée Infection, Marseille, France. ${ }^{3}$ ALTRABIO, Lyon, France.

Correspondence: Alain Boussuges, C2VN, Center for Cardiovascular and Nutrition Research, Aix-Marseille Université et Institut de Recherche Biomédicale des Armées (IRBA), Faculté de Médecine de Marseille, 27 Boulevard Jean Moulin, 13385 Marseille Cedex 05, France. E-mail: alain.boussuges@univ-amu.fr

ABSTRACT Chest ultrasonography has proven to be useful in the diagnosis of diaphragm dysfunction. The aim of the present study was to determine the normal values of the motion of both hemidiaphragms recorded by M-mode ultrasonography.

Healthy volunteers were studied while in a seated position. Diaphragmatic excursions and diaphragm profiles were measured during quiet breathing, voluntary sniffing and deep breathing. Diaphragmatic excursions were assessed by M-mode ultrasonography, using an approach perpendicular to the posterior part of the diaphragm. Anatomical M-mode was used for the recording of the complete excursion during deep breathing.

The study included 270 men and 140 women. The diaphragmatic motions during quiet breathing and voluntary sniffing were successfully recorded in all of the participants. The use of anatomical M-mode was particularly suitable for measurement of the entire diaphragmatic excursion during deep breathing. The statistical analysis showed that the diaphragmatic excursions were larger in men compared to women, supporting the determination of normal values based on sex. The lower and upper limits of normal excursion were determined for men and women for both hemidiaphragms during the three manoeuvres that were investigated. The lower limits of normal diaphragmatic excursions during deep breathing should be used to detect diaphragmatic hypokinesia, i.e. 3.3 and $3.2 \mathrm{~cm}$ in women and 4.1 and $4.2 \mathrm{~cm}$ in men for the right and the left sides, respectively.

The normal values of the diaphragmatic motion and the lower and upper limits of normal excursion can be used by clinicians to detect diaphragmatic dysfunction.

@ERSpublications

These normal values of diaphragmatic motion, and the lower and upper limits of normal excursion, can be used by physicians to detect diaphragm hypokinesia and hyperkinesia, and thus diagnose diaphragmatic dysfunction https://bit.ly/35R9OFk

Cite this article as: Boussuges A, Finance J, Chaumet G, et al. Diaphragmatic motion recorded by M-mode ultrasonography: limits of normality. ERJ Open Res 2021; 7: 00714-2020 [https://doi.org/ 10.1183/23120541.00714-2020].

Received: 1 Oct 2020 | Accepted: 4 Jan 2021

Copyright $\odot$ The authors 2021. This version is distributed under the terms of the Creative Commons Attribution NonCommercial Licence 4.0. For commercial reproduction rights and permissions contact permissions@ersnet.org 


\section{Introduction}

Chest ultrasonography has proven to be useful in the diagnosis of diaphragmatic paralysis. The criteria for the diagnosis of diaphragmatic paralysis by M-mode ultrasonography have been reported previously $[1,2]$. The study of diaphragmatic motion has demonstrated its relevance in various conditions such as after thoracic surgery [3] and in patients suffering from neuromuscular diseases [4, 5].

On the other hand, there is no clear definition of diaphragmatic dysfunction in the literature. In the absence of a noninvasive reference diagnostic tool to ascertain diaphragm dysfunction, a diagnostic approach can be used based on the motion and thickness changes of both hemidiaphragms during inspiration. Providing robust reference values from healthy subjects for diaphragmatic motion is, therefore, of major interest.

To detect diaphragmatic dysfunction, a number of previous studies $[6,7]$ have researched the values corresponding to the lower limit of normality (LLN) for diaphragmatic excursions. When the examination reveals excursion of one hemidiaphragm lower than the LLN but no paradoxical motion, hemidiaphragm dysfunction without complete paralysis can be suspected. Consequently, ultrasonography may be useful as a screening test in patients suffering from various diseases known to impair diaphragmatic function such as neuromuscular diseases [8].

The normal values of diaphragmatic excursions studied by M-mode ultrasonography [9-11] have most often been based on studies of subjects in the supine position (for a review see [12]). It has been reported that for the same volume inspired, excursions are larger in the supine position than with standing or sitting positions [13]. It is, therefore, important to use different normal values according to the position of the subject. In contrast to a seated position, supine and standing positions are not well tolerated by some subjects, such as patients suffering from respiratory problems. Consequently, it is important to determine the normal values of diaphragmatic excursions in subjects assessed while in a seated position. Two studies have used a semi-recumbent position in a small sample of healthy volunteers (64 volunteers for CARDENAS et al. [7], 40 for TESTA et al. [14]). For a number of reasons, the populations studied did not appear to be sufficient to determine robust reference values.

As sex is known to impact diaphragmatic motion [6-9], it is important to study a large population to determine appropriate normal values and LLN according to sex.

Furthermore, in nearly the entire population of 210 participants in the study by Boussuges et al. [6], the excursions of the two hemidiaphragms were recorded during rest breathing and voluntary sniffing. In contrast, the excursions during deep breathing were measured on the right side in 195 and on the left side in 45 subjects. The recordings of the diaphragmatic excursions during this manoeuvre were compromised because the hemidiaphragms were frequently obscured by the descending lung. Thus, a large population should be used to increase the accuracy of the LLN during deep breathing. Lastly, various respiratory diseases have an impact on the diaphragmatic pattern as a result of an increase in the inspiratory and expiratory work of breathing $[15,16]$. The normal values of both the contraction and relaxation profiles should be determined before further studies are carried out on this topic.

The present study was designed to determine the normal values of diaphragmatic motion, including the excursions and the profiles of the contraction and relaxation, recorded in a healthy population assessed while in a sitting position.

\section{Methods}

A weekly medical consultation was conducted from January, 2018, to January, 2020, to recruit healthy volunteers. The Ethics Committee of the Aix-Marseille University (CPPRB 1, NoA01299-32) approved the study protocol, and written consent was obtained from all of the volunteers. Volunteers were considered to be healthy when they did not have a history of respiratory disorders, they had no clinical issues at the time of the examination and they had a normal pulmonary function test. Pulmonary function was studied with a spirometer (Ilmeter 1304; Masterlab Jaeger, Würzberg, Germany) according to the European Respiratory Society (ERS)/American Thoracic Society (ATS) standards [17]. The criteria for classifying the pulmonary function test as normal were a slow vital capacity (SVC), a forced vital capacity (FVC), and a forced expiratory volume in $1 \mathrm{~s}\left(\mathrm{FEV}_{1}\right)$ larger than the LLN for the reference population, and a $\mathrm{FEV}_{1}$ to FVC ratio $>0.7$.

In a previous study [6] we reported that when the examinations were performed in a standing position, the diaphragmatic excursions were greater in healthy men $(1.8 \pm 0.4 \mathrm{~cm})$ compared to healthy women $(1.6 \pm 0.4 \mathrm{~cm})$. To find differences between the sexes and appropriate normal values for men and women, the calculation of the sample was based on this previous study. For a $0.05 \% \alpha$ risk and a $95 \%$ power, we determined that 105 volunteers would have to be included in each group (men and women). Since some 
parameters, such as the excursion during deep breathing, were sometimes impossible to record in our previous study [6], it was decided to increase the study population. For this reason, we aimed for a sample size of $>125$ individuals in both groups.

The ultrasonographic examinations were carried out by two experienced investigators (both investigators had performed $>300$ ultrasound examinations of the diaphragm before the beginning of the study) using a commercially available Doppler echocardiograph machine (Vivid S60N, GE Medical System, Milwaukee, WI, USA) connected to a $2-4.5 \mathrm{MHz}$ transducer array (3Sc probe).

The patients were placed in a sitting position and the diaphragmatic excursions were assessed by M-mode ultrasonography according to a previously published method [6].

A subcostal or low intercostal probe position was chosen between the midclavicular and posterior axillary lines to obtain the best imaging of both hemidiaphragmatic domes using two-dimensional mode (B-mode). When the approach of the hemidiaphragm was considered to be good and the exploration line was perpendicular to the posterior part of each hemidiaphragm, M-mode was used to record the diaphragmatic motion. The diaphragmatic motion was assessed by M-mode while the patient breathed on tidal volume (quiet breathing). Furthermore, the diaphragmatic excursions were measured during voluntary sniffing and deep breathing (from the functional residual capacity to the total lung capacity).

Recordings of the right hemidiaphragm motion were obtained by placing the ultrasonography probe on the subcostal or low intercostal area, between the midclavicular and the mean axillary lines. For the left side, the probe had to be in a more posterior position than on the right side (i.e. between the anterior and the posterior axillary lines).

During deep breathing, the dome was frequently obscured by the descending lung. To improve the recording of the entire excursion, a caudal displacement of the probe associated with angulation to maintain the approach perpendicular to the posterior part of the diaphragm was performed. Nevertheless, the recording of the complete excursion could still be problematic and the angle of the approach of the hemidiaphragm was frequently far from perpendicular. In these circumstances, anatomical M-mode was used (figure 1). All of the examinations were recorded for subsequent blind analysis.

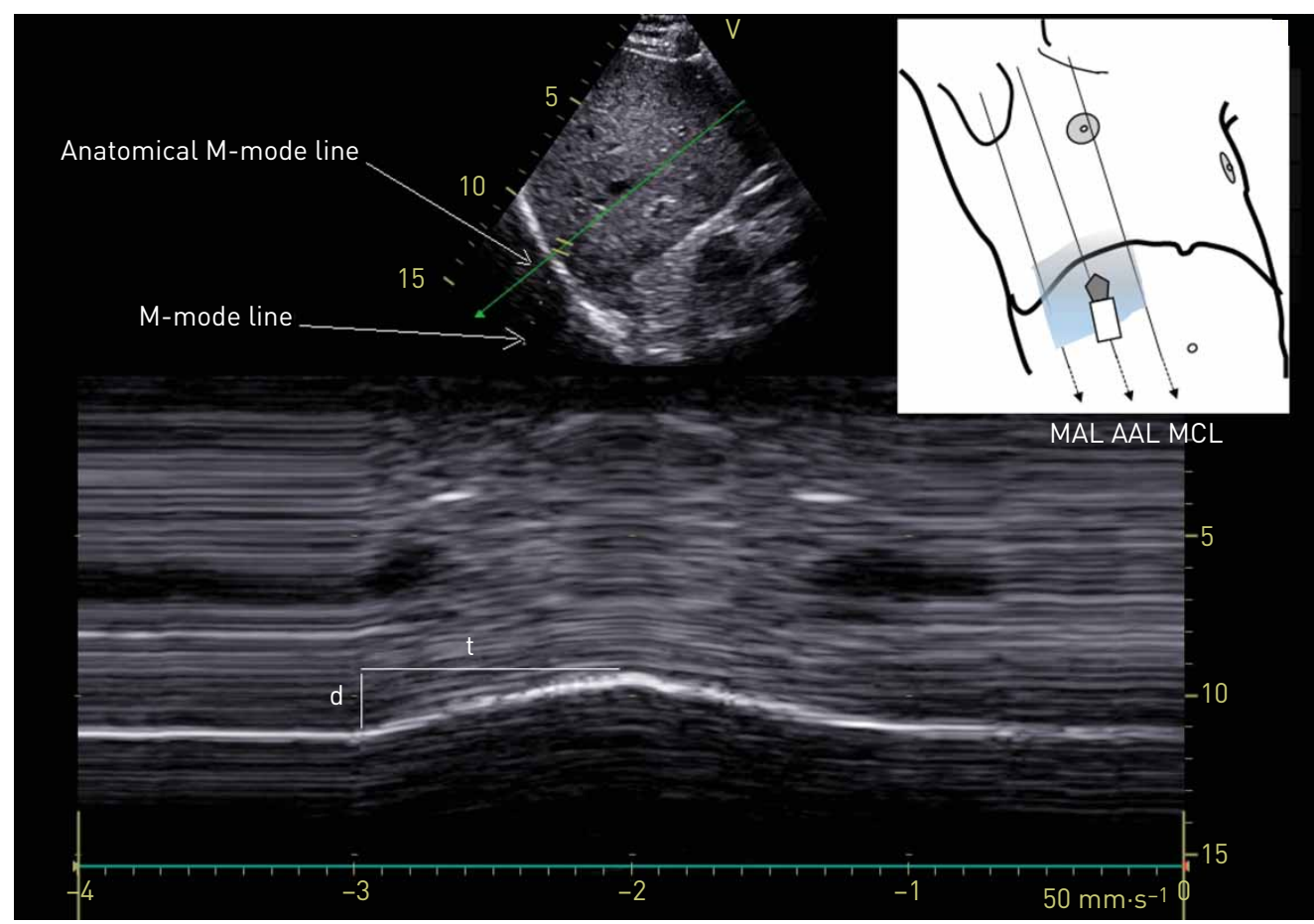

FIGURE 1 Right hemidiaphragmatic motion recorded by anatomical M-mode ultrasonography. The anatomical $\mathrm{M}$-mode cursor was rotated and placed on the exact axis of the diaphragm displacement. Measurement of the diaphragm excursion (d) and the inspiratory time $(t)$ were carried out on the diaphragmatic displacement recorded along the anatomical M-mode line; the velocity of contraction was calculated as d/t. Inset: for the recording of the right hemidiaphragmatic motion, the probe was placed between the midclavicular line and the mean axillary line. MAL: mean axillary line; AAL: anterior axillary line; MCL: midclavicular line. 


\section{Measurements}

The diaphragmatic inspiratory excursions were measured by placing the first caliper at the foot of the inspiration slope on the diaphragmatic echoic line and by placing the second caliper at the apex of the curve. For the rest breathing, the times and the velocities of displacement (the slopes) of both the inspiratory and the expiratory phases were also measured. The measurements of at least three different breathing cycles were then averaged. For voluntary sniffing, the excursion, the velocity and the duration of the displacement were measured. For deep breathing, several manoeuvres were recorded, and the maximal excursion, i.e. the largest distance between the baseline and the apex, was retained.

\section{Statistical analysis}

The results are reported as mean \pm SD. The lower and upper limits of normal were calculated as mean \pm 1.95 SD. The statistical analyses were performed using R statistical software [18]. A linear regression analysis was performed to assess how the ultrasonographic measurements varied according to sex, age and body mass index (BMI).

\section{Results}

\section{Population studied}

410 healthy volunteers ( 270 men and 140 women) were included in the study. Their mean age was $52 \pm 16$ years, their mean height was $166 \pm 18 \mathrm{~cm}$ and their mean weight was $75 \pm 22 \mathrm{~kg}$. Their pulmonary function tests were normal, with a mean SVC of $4.1 \pm 1.1 \mathrm{~L}(102 \pm 14 \%$ pred), a mean FVC of $3.8 \pm 1.1 \mathrm{~L}(97 \pm 14 \%$ pred) and a mean $\mathrm{FEV}_{1}$ of $3.1 \pm 0.9 \mathrm{~L}(98 \pm 13 \%$ pred). The demographic data of the men were: age $52 \pm 15$ years, height $173 \pm 7 \mathrm{~cm}$, weight $76 \pm 12 \mathrm{~kg}$ and BMI $25 \pm 4 \mathrm{~kg} \cdot \mathrm{m}^{-2}$. The demographic data of the women were: age $52 \pm 16$ years, height $159 \pm 14 \mathrm{~cm}$, weight $66 \pm 18 \mathrm{~kg}$ and BMI $25 \pm 5 \mathrm{~kg} \cdot \mathrm{m}^{-2}$.

\section{Ultrasonographic recordings}

The diaphragmatic motion during quiet breathing and voluntary sniffing was successfully recorded in all of the volunteers.

For the recording of the entire excursion during deep breathing, at the beginning of the study period in the first series of 100 patients, when the caudal displacement of the probe and the anatomical M-mode were not used, the right and the left hemidiaphragm excursions could be measured in 97 and 43 of cases, respectively. The use of caudal probe displacement and anatomical M-mode allowed for a greater degree of success with recording the diaphragmatic motion during deep breathing. Indeed, in the last 100 patients measured, the recording of the diaphragmatic excursions during deep breathing was successful in all of the volunteers on the right side and in $96 \%$ of cases on the left side. This finding was reported although the demographic data were similar in the two groups. In the whole population, the diaphragmatic excursion during deep breathing could be successfully measured in $98 \%$ of cases on the right side and in $56 \%$ of cases on the left side.

Tables 1 and 2 list the results of the diaphragmatic motion during quiet breathing, voluntary sniffing and deep breathing on the right side and the left side, respectively. The statistical analysis revealed larger excursions in men compared to women on both sides and for the three manoeuvres studied, i.e. quiet breathing, voluntary sniffing and deep breathing. The right-to-left ratio with quiet breathing and with deep breathing was around 1 , and it was similar for both sexes.

For the multivariate regression analyses, patients with missing data in the variable to be explained were removed. The results of the linear regression analysis are reported in table 3; the number of patients for each analysis is indicated in the last column.

\section{Discussion}

Our study reports the normal values of diaphragmatic motion recorded during quiet breathing, voluntary sniffing and deep breathing in a large population of healthy volunteers of both sexes assessed while in a seated position. The lower limits for normal excursions of both hemidiaphragms reported in the present study can be used to detect hypokinesia, which is indicative of diaphragmatic dysfunction.

Our work was based on assessment of a large population with the aim of improving the accuracy of the lower and upper limits of normal excursion during quiet breathing, voluntary sniffing and deep breathing. The statistical analysis supports the previous finding that the normal values depend on sex, since the excursions measured in men were larger than those in women (see tables 1 and 2). A number of previous studies performed in the supine position have reported an impact of age on the diaphragmatic excursion measured during deep inspiration. A positive correlation was found by KANTARCI et al. [9], whereas a negative correlation was reported by SCARLATA et al. [10]. We found that age did not have an impact on the diaphragmatic excursion. On the other hand, as previously reported in several studies [6-10], the impact 


\begin{tabular}{|c|c|c|c|}
\hline & Women & Men & p-value \\
\hline \multicolumn{4}{|l|}{ Quiet breathing } \\
\hline Excursion $\mathrm{cm}$ & $1.7 \pm 0.4(0.9-2.5)$ & $1.9 \pm 0.5(0.9-2.8)$ & $<0.01$ \\
\hline Inspiratory time s & $1.1 \pm 0.3(0.5-1.7)$ & $1.1 \pm 0.4(0.4-1.8)$ & NS \\
\hline Inspiratory velocity $\mathrm{cm} \cdot \mathrm{s}^{-1}$ & $1.7 \pm 0.5(0.7-2.6)$ & $1.8 \pm 1.1(0-4)$ & NS \\
\hline Expiratory time $s$ & $1 \pm 0.3(0.4-1.6)$ & $1.1 \pm 0.4(0.4-1.8)$ & $<0.05$ \\
\hline Expiratory velocity $\mathrm{cm} \cdot \mathrm{s}^{-1}$ & $1.5 \pm 0.7(0.2-2.8)$ & $1.5 \pm 0.6(0.4-2.7)$ & NS \\
\hline Duration of motion $\mathrm{s}$ & $2.3 \pm 0.6(1.1-3.4)$ & $2.4 \pm 0.7(1-3.8)$ & $<0.05$ \\
\hline Right-to-left ratio with quiet breathing & $1.1 \pm 0.3(0.5-1.6)$ & $1 \pm 0.3(0.4-1.5)$ & NS \\
\hline \multicolumn{4}{|l|}{ Voluntary sniffing } \\
\hline Excursion $\mathrm{cm}$ & $2.6 \pm 0.6(1.3-3.9)$ & $2.9 \pm 0.7(1.5-4.3)$ & $<0.001$ \\
\hline Inspiratory time s & $0.3 \pm 0.1(0.1-0.5)$ & $0.3 \pm 0.1(0.1-0.5)$ & NS \\
\hline Inspiratory velocity $\mathrm{cm} \cdot \mathrm{s}^{-1}$ & $9.9 \pm 3.5(3-16.7)$ & $12 \pm 4.9(2.5-21.8)$ & $<0.001$ \\
\hline \multicolumn{4}{|l|}{ Deep breathing } \\
\hline Excursion $\mathrm{cm}$ & $5.4 \pm 1.1(3.3-7.5)$ & $6.6 \pm 1.3(4.1-9)$ & $<0.001$ \\
\hline Inspiratory time s & $1.4 \pm 0.5(0.4-2.4)$ & $1.4 \pm 0.6(0.2-2.6)$ & NS \\
\hline Inspiratory velocity $\mathrm{cm} \cdot \mathrm{s}^{-1}$ & $4.4 \pm 1.8(1.5-7.3)$ & $5.5 \pm 2.7(0.2-10.9)$ & $<0.001$ \\
\hline Right-to-left ratio with deep breathing & $1 \pm 0.2(0.6-1.4)$ & $1 \pm 0.2(0.6-1.3)$ & NS \\
\hline
\end{tabular}

Data are presented as mean \pm SD (lower limit of normal - upper limit of normal), unless otherwise stated. NS: nonsignificant.

of anthropometric data on the diaphragmatic motion is supported, in our results, by the positive correlations between the BMI and the diaphragmatic excursions (see table 3).

The mean values and the lower limits of normal diaphragmatic excursion during quiet breathing and voluntary sniffing are close $( \pm 1 \mathrm{~mm})$ to the values previously reported for standing healthy volunteers [6]. By contrast, the present work reports lower mean values and thresholds for the excursions recorded during deep breathing, thus suggesting that the diaphragmatic excursions during this manoeuvre are larger with a standing than with a sitting position. Furthermore, it is generally recognised that when the approach to the hemidiaphragm by M-mode is not perpendicular to the cranial-caudal motion, the displacement can be overestimated through the sum of two errors: an orientation error (the M-mode plane is not orientated to the true direction of the diaphragm displacement) and a translation error (adjacent closer portions of the diaphragm enter the M-mode plane of interrogation) $[19,20]$. In the present work, special care was taken to reach the hemidiaphragm in a perpendicular manner. The benefit of anatomical M-mode is clear

TABLE 2 Left diaphragmatic motion and limit values in women and men

\begin{tabular}{|c|c|c|c|}
\hline & Women & Men & p-value \\
\hline \multicolumn{4}{|l|}{ Quiet breathing } \\
\hline Excursion $\mathrm{cm}$ & $1.7 \pm 0.4(0.9-2.5)$ & $2 \pm 0.6(0.9-3)$ & $<0.001$ \\
\hline Inspiratory time s & $1 \pm 0.2(0.5-1.4)$ & $1 \pm 0.3(0.5-1.6)$ & $<0.01$ \\
\hline Inspiratory velocity $\mathrm{cm} \cdot \mathrm{s}^{-1}$ & $1.9 \pm 0.6(0.7-3)$ & $2 \pm 0.8(0.4-3.6)$ & NS \\
\hline Expiratory time $\mathrm{s}$ & $0.8 \pm 0.3(0.3-1.4)$ & $0.9 \pm 0.3(0.2-1.5)$ & NS \\
\hline Expiratory velocity $\mathrm{cm} \cdot \mathrm{s}^{-1}$ & $1.8 \pm 0.7(0.5-3.1)$ & $2.1 \pm 0.9(0.4-3.7)$ & $<0.001$ \\
\hline Duration of motion s & $2 \pm 0.5(1-3)$ & $2.2 \pm 0.7(0.9-3.5)$ & $<0.01$ \\
\hline \multicolumn{4}{|l|}{ Voluntary sniffing } \\
\hline Excursion $\mathrm{cm}$ & $2.6 \pm 0.6(1.5-3.8)$ & $3 \pm 0.8(1.6-4.5)$ & $<0.001$ \\
\hline Inspiratory time s & $0.2 \pm 0.1(0.1-0.4)$ & $0.2 \pm 0.1(0.1-0.4)$ & NS \\
\hline Inspiratory velocity $\mathrm{cm} \cdot \mathrm{s}^{-1}$ & $12.3 \pm 4.4(3.6-20.9)$ & $15 \pm 7.6(1.2-30)$ & $<0.001$ \\
\hline \multicolumn{4}{|l|}{ Deep breathing } \\
\hline Excursion $\mathrm{cm}$ & $5.4 \pm 1.2(3.2-7.7)$ & $6.7 \pm 1.3(4.2-9.2)$ & $<0.001$ \\
\hline Inspiratory time s & $1.2 \pm 0.5(0.2-2.3)$ & $1.1 \pm 0.4(0.26-2)$ & NS \\
\hline Inspiratory velocity $\mathrm{cm} \cdot \mathrm{s}^{-1}$ & $5.3 \pm 2.5(0.4-10)$ & $6.6 \pm 2.6(1.6-11.6)$ & $<0.001$ \\
\hline
\end{tabular}

Data are presented as mean \pm SD (lower limit of normal - upper limit of normal), unless otherwise stated. NS: nonsignificant. 


\begin{tabular}{|c|c|c|c|c|}
\hline Manoeuvre & Side & Term & p-value & $\mathbf{n}$ \\
\hline \multirow[t]{6}{*}{ Quiet breathing } & \multirow[t]{3}{*}{ Left } & Sex (male) & $<0.05$ & \multirow[t]{3}{*}{410} \\
\hline & & Age & NS & \\
\hline & & BMI & $<0.05$ & \\
\hline & \multirow[t]{3}{*}{ Right } & Sex (male) & 0.01 & \multirow[t]{3}{*}{410} \\
\hline & & Age & NS & \\
\hline & & $\mathrm{BMI}$ & $<0.001$ & \\
\hline \multirow[t]{6}{*}{ Voluntary sniffing } & \multirow[t]{3}{*}{ Left } & Sex (male) & $<0.0001$ & \multirow[t]{3}{*}{410} \\
\hline & & Age & NS & \\
\hline & & BMI & NS & \\
\hline & \multirow[t]{3}{*}{ Right } & Sex (male) & 0.01 & \multirow[t]{3}{*}{410} \\
\hline & & Age & NS & \\
\hline & & BMI & NS & \\
\hline \multirow[t]{6}{*}{ Deep breathing } & \multirow[t]{3}{*}{ Left } & Sex (male) & $<0.0001$ & \multirow[t]{3}{*}{231} \\
\hline & & Age & NS & \\
\hline & & BMI & $<0.01$ & \\
\hline & \multirow[t]{3}{*}{ Right } & Sex (male) & $<0.0001$ & \multirow[t]{3}{*}{403} \\
\hline & & Age & NS & \\
\hline & & BMI & $<0.05$ & \\
\hline
\end{tabular}

since the percentage of successful recordings of the entire excursion of the hemidiaphragm was dramatically increased when this modality was used, particularly on the left side. Our results are in keeping with previous studies that reported measurement of the left hemidiaphragm motion in $>90 \%$ of cases using anatomical M-mode [20,21]. Consequently, the threshold values reported in our work should be more accurate than those in previous studies.

New upper limit values of normal excursion were also determined. These values should be of considerable value to investigators. Indeed, large excursions on the healthy side during quiet breathing have been frequently reported in patients suffering from hemidiaphragm paralysis [2-22]. These large excursions have been attributed to a compensatory mechanism including an increase in neural drive to the functioning hemidiaphragm [23].

Comparative assessment of diaphragmatic displacement of both sides during deep breathing has been proposed to detect unilateral diaphragmatic dysfunction. Houston et al. [24] have determined a normal range for the right-to-left ratio of maximal excursion on deep breathing. They considered that a right-to-left ratio outside of a range of 0.5 to 1.6 should be deemed to be abnormal. In our study, the mean ratio was near unity for both sexes and close to the lower and upper limit threshold values proposed by Houston et al. [24], i.e. 0.6 to 1.3 for men and 0.6 to 1.4 for women. Thus, our results are further indication of the relevance of comparison of the two hemidiaphragm excursions. A ratio of displacement outside these limits is very much a reason to suspect abnormal motion.

It is recognised that an obstructive lesion of the upper airway induces abnormalities in the pattern of resting breathing such as an increase in the inspiratory time over total time and a decrease in the inspiratory airflow [15]. Changes in the diaphragmatic motion profile can be expected in such circumstances. Indeed, alteration of the diaphragmatic motion recorded by M-mode ultrasonography has been reported in healthy individuals subjected to an increase in inspiratory resistance [25]. A decrease in the breathing rate and an increase in both the diaphragmatic excursion and the inspiratory time were shown to occur, compared to breathing without respiratory load [25]. Consequently, the normal values of the profile of the contraction of the hemidiaphragms should be useful for assessment of the impact of an upper airway obstruction such as a tracheal stenosis. The normal values of duration (mean $1.1 \mathrm{~s}$ ) and velocity of inspiratory time (mean $1.7 \mathrm{~cm} \cdot \mathrm{s}^{-1}$ ) of the right diaphragmatic motion, reported in our study during quiet breathing, were close to the results of Ayoub et al. [26] and SPIESSHOefer et al. [27].

Our study reports the normal values of diaphragmatic motion recorded in a population of healthy volunteers assessed while in a seated position. These results may be of limited relevance for subjects assessed in other positions or with health conditions. Nevertheless, when a mouthpiece is used to measure the volume of gas of a patient during the ultrasound examination, the increase in resistance leads to an 
increase in diaphragmatic excursions $[25,26]$. Since diaphragmatic excursions are thought to be increased in patients wearing a mouthpiece and when they are in the supine position, in such circumstances, when an excursion is lower than the LLN determined in our study, an abnormal motion can be strongly suspected.

When a patient can tolerate both a seated and the supine position, it can be useful to repeat the ultrasound examination in these two conditions, especially when diaphragmatic motion appears to be impaired. Indeed, it has been reported that in the supine position, the abdominal visceral mass increased diaphragmatic work and that the compensatory active expiration by the anterior abdominal wall was decreased [28]. Consequently, in patients suffering from hemidiaphragm paralysis, paradoxical movements could be unmasked in the supine position. Lastly, it has been reported that diaphragmatic function can be assessed using measurement of the diaphragm thickness at the zone of apposition of the diaphragm to the rib cage [29]. In patients suffering from hemidiaphragm paralysis, on the paralysed side the hemidiaphragm did not thicken significantly or it even became thinner [30]. A threshold of $20 \%$ is accepted by most authors for the diagnosis of hemidiaphragm paralysis [12]. In patients with hemidiaphragm excursions lower than the LLN, the control of diaphragmatic motion in the supine position and the measurement of inspiratory diaphragm thickening provide information in support of a diagnosis of diaphragmatic dysfunction.

Acknowledgements: The authors gratefully acknowledge the volunteers. Alain Boussuges takes responsibility for the content of the manuscript, including the data and analysis.

Author contributions: A. Boussuges conceived and designed the study. F. Brégeon and J. Finance assisted with the technical aspects of the study and were involved in the acquisition of the data. A. Boussuges and G. Chaumet analysed the data and performed the statistical analysis. A. Boussuges and G. Chaumet drafted the article, while F. Brégeon critically revised it for its main intellectual content. All of the authors approved the final version of the manuscript. All of the persons designated as authors qualify for authorship, and all those who qualify for authorship are listed.

Conflict of interest: None declared.

\section{References}

1 Lloyd T, Tang YM, Benson MD, et al. Diaphragmatic paralysis: the use of M mode ultrasound for diagnosis in adults. Spinal Cord 2006; 44: 505-508.

2 Boussuges A, Brégeon F, Blanc P, et al. Characteristics of the paralysed diaphragm studied by M-mode ultrasonography. Clin Physiol Funct Imaging 2019; 39: 143-149.

3 Spadaro S, Grasso S, Dres M, et al. Point of care ultrasound to identify diaphragmatic dysfunction after thoracic surgery. Anesthesiology 2019; 131: 266-278.

4 Minami T, Manzoor K, McCool FD. Assessing diaphragm function in chest wall and neuromuscular diseases. Clin Chest Med 2018; 39: 335-344.

5 Fayssoil A, Nguyen LS, Ogna A, et al. Diaphragm sniff ultrasound: normal values, relationship with sniff nasal pressure and accuracy for predicting respiratory involvement in patients with neuromuscular disorders. PLoS One 2019; 14: e0214288.

6 Boussuges A, Gole Y, Blanc P. Diaphragmatic motion studied by M-mode ultrasonography: methods, reproducibility, and normal values. Chest 2009; 135: 391-400.

7 Cardenas LZ, Santana PV, Caruso P, et al. Diaphragmatic ultrasound correlates with inspiratory muscle strength and pulmonary function in healthy subjects. Ultrasound Med Biol 2018; 44: 786-793.

8 Carrié C, Bonnardel E, Vally R, et al. Vital capacity impairment due to neuromuscular disease and its correlation with diaphragmatic ultrasound: a preliminary study. Ultrasound Med Biol 2016; 42: 143-149.

9 Kantarci F, Mihmanli I, Demirel MK, et al. Normal diaphragmatic motion and the effects of body composition: determination with M-mode sonography. J Ultrasound Med 2004; 23: 255-260.

10 Scarlata S, Mancini D, Laudisio A, et al. Reproducibility and clinical correlates of supine diaphragmatic motion measured by M-mode ultrasonography in healthy volunteers. Respiration 2018; 96: 259-266.

11 Katipoglu B, Kuday Kaykisiz E, Szarpak L, et al. The evaluation of reference values for diaphragmatic excursion in Turkish population. Eurasian J Critical Care 2019; 1: 1-6.

12 Boussuges A, Rives S, Finance J, et al. Assessment of diaphragmatic function by ultrasonography: current approach and perspectives. World J Clin Cases 2020; 8: 2408-2424.

13 Houston JG, Angus RM, Cowan MD, et al. Ultrasound assessment of normal hemidiaphragmatic movement: relation to inspiratory volume. Thorax 1994; 49: 500-503.

14 Testa A, Soldati G, Giannuzzi R, et al. Ultrasound M-mode assessment of diaphragmatic kinetics by anterior transverse scanning in healthy subjects. Ultrasound Med Biol 2011; 37: 44-52.

15 Sanchis J, Diez-Bethoret JL, Casan P, et al. The pattern of resting breathing in patients with upper airway obstruction. Eur Respir J 1990; 3: 521-526.

16 Pellegrino R, Brusasco V. Lung hyperinflation and flow limitation in chronic airway obstruction. Eur Respir $J$ 1997; 10: 543-549.

17 Graham BL, Steenbruggen I, Miller MR, et al. Standardization of Spirometry 2019 Update. An Official American Thoracic Society and European Respiratory Society Technical Statement. Am J Respir Crit Care Med 2019; 200: e70-e88.

18 Lagani V, Athineou G, Farcomeni A, et al. Feature selection with the R package MXM: discovering statistically-equivalent feature subsets. J Statistical Software 2017; 80: 1-25. 
19 Orde SR, Boon AJ, Firth DG, et al. Use of angle-independent M-mode sonography for assessment of diaphragm displacement. J Ultrasound Med 2016; 35: 2615-2621.

20 Goutman SA, Hamilton JD, Rubin JM. Use of angle-independent M-Mode sonography for assessment of diaphragm displacement. J Ultrasound Med 2017; 36: 1285.

21 Pasero D, Koeltz A, Placido R, et al. Improving ultrasonic measurement of diaphragmatic excursion after cardiac surgery using the anatomical M-mode: a randomized crossover study. Intensive Care Med 2015; 41: 650-656.

22 Houston JG, Fleet M, Cowan MD, et al. Comparison of ultrasound with fluoroscopy in the assessment of suspected hemidiaphragmatic movement abnormality. Clin Radiol 1995; 50: 95-98.

23 Katagiri M, Young RN, Platt RS, et al. Respiratory muscle compensation for unilateral or bilateral hemidiaphragm paralysis in awake canines. J Appl Physiol 1994; 77: 1972-1982.

24 Houston JG, Morris AD, Howie CA, et al. Technical report: quantitative assessment of diaphragmatic movement: a reproducible method using ultrasound. Clin Radiol 1992; 46: 405-407.

25 Soilemezi E, Tsagourias M, Talias MA, et al. Sonographic assessment of changes in diaphragmatic kinetics induced by inspiratory resistive loading. Respirology 2013; 18: 468-473.

26 Ayoub J, Cohendy R, Dauzat M, et al. Non-invasive quantification of diaphragm kinetics using M-mode sonography. Can J Anaesth 1997; 44: 739-744.

27 Spiesshoefer J, Herkenrath S, Henke C, et al. Evaluation of respiratory muscle strength and diaphragm ultrasound: normative values, theoretical considerations, and practical recommendations. Respiration 2020; 99: 369-381.

28 McCool FD, Mead J. Dyspnea on immersion: mechanisms in patients with bilateral diaphragm paralysis. Am Rev Respir Dis 1989; 139: 275-276.

29 Boon AJ, Sekiguchi H, Harper CJ, et al. Sensitivity and specificity of diagnostic ultrasound in the diagnosis of phrenic neuropathy. Neurology 2014; 83: 1264-1270.

30 Gottesman E, McCool FD. Ultrasound evaluation of the paralyzed diaphragm. Am J Respir Crit Care Med 1997; 155: $1570-1574$ 